\title{
The role of reconnection in the pulsar magnetosphere
}

\author{
I. Contopoulos
}

\author{
Research Center for Astronomy, Academy of Athens, 11527 Athens, Greece \\ e-mail: icontop@academyofathens.gr
}

Received 5 July 2006 / Accepted 2 October 2006

\section{ABSTRACT}

\begin{abstract}
The present work is our first attempt to understand the role of reconnection in the pulsar magnetosphere. Our discussion is based on the observational inference that, as the pulsar spins down, the region of closed corotating dipolar field lines grows with time. This implies that reconnection must take place in the magnetosphere. We argue that non-dissipative reconnection along the equatorial current sheet allows for continuous channeling of pulsar spindown energy into particle energy, all the way from the light cylinder to the pulsar wind termination shock, and we propose that this effect may account for the low $\sigma_{\text {shock }}$ values inferred from the observations. We present a simple model that allows us to relate the magnetic diffusivity in the equatorial current sheet to an observable pulsar parameter, the braking index $n$. When $n \sim 1$, the global structure of the magnetosphere approaches that of a relativistic split monopole where the pulsar spindown energy is carried by the electromagnetic field. However, for values of $n \gtrsim 1.5$, almost all field lines close inside the pulsar wind termination shock, and thus most of the electromagnetic pulsar spindown energy flux is effectively transformed into particle energy in the equatorial current sheet.
\end{abstract}

Key words. pulsars: general - magnetic fields - magnetohydrodynamics (MHD)

\section{Introduction}

In the past few years, several steps have been taken that improved our understanding of the electrodynamic structure of the pulsar magnetosphere (Michel 1991; Contopoulos et al. 1999, hereafter CKF; Mestel 1999; Bogovalov 1999; Spitkovsky 2006). A canonical paradigm begins to emerge, namely that a magnetized rotating neutron star with polar magnetic field $B_{*} \sim 10^{13} \mathrm{G}$, mass $M_{*} \sim 1.4 M_{\odot}$, radius $r_{*} \sim 10 \mathrm{~km}$, and angular velocity $\Omega$ loses rotational kinetic energy at a rate of

$\dot{E}_{\mathrm{kin}}=\frac{2}{5} M_{*} r_{*}^{2} \Omega \dot{\Omega}$,

through electromagnetic torques in its magnetosphere. The poloidal structure of the pulsar magnetosphere remains dipolarlike (closed) from the surface of the rotating central neutron star to a distance

$r_{\text {open }} \leq r_{\mathrm{lc}}=\frac{c}{\Omega}$,

where $r_{\mathrm{lc}}$ is the so-called "light cylinder". Beyond $r_{\mathrm{open}}$, the poloidal structure of the magnetosphere becomes monopole-like (open). The total amount of open poloidal magnetic flux is on the order of

$\Psi_{\text {open }}=1.23 \frac{\pi B_{*} r_{*}^{3}}{r_{\text {open }}}$.

The magnetic field along the open monopole-like magnetic field lines is wound backwards in the azimuthal direction by the neutron star rotation, and a toroidal magnetic field is generated. The source of the toroidal field is a poloidal electric current whose distribution along the open field lines is given by

$I(\Psi) \approx-\frac{\Omega \Psi}{4 \pi}\left(2-\frac{\Psi}{\Psi_{\text {open }}}\right)$, when $0 \leq \Psi \leq \Psi_{\text {open }}$, and zero outside. This current distribution implies the existence of a singular return electric current sheet flowing along the equator and along the separatrix between open and closed field lines. Beyond distances greater than a few times the light cylinder distance, the dominant field component becomes the azimuthal one. Finally, the electromagnetic spindown luminosity is given by

$L=\frac{\Omega^{2} \Psi_{\mathrm{open}}^{2}}{6 \pi^{2} c}$.

Note that Eqs. (3)-(5) are valid for an aligned rotator. The case of an oblique rotator remains under investigation (Bogovalov 1999; Spitkovsky 2006; Contopoulos 2006). We have introduced here a cylindrical system of coordinates $(r, z, \phi)$ aligned with the central rotating neutron star; we also introduced the magnetic flux $\Psi$ as

$\boldsymbol{B}_{\mathrm{p}} \equiv \nabla \times\left(\frac{\Psi}{2 \pi r} \hat{\phi}\right)$,

where $\boldsymbol{B}_{\mathrm{p}}$ is the poloidal component of the magnetic field. Furthermore, $(\ldots) \equiv \partial(\ldots) / \partial t$.

In developing the above paradigm, there has been almost universal agreement within the astrophysics community that the global structure of the pulsar magnetosphere may be described by the ideal MHD formalism, except for regions of finite extent, such as regions of particle acceleration along the magnetic field (polar gaps (e.g. Daugherty \& Harding 1982), slot gaps (Harding \& Muslimov 2005), outer gaps (e.g. Romani \& Yadigaroglu 1995), light cylinder dissipation regions (e.g. Mestel \& Shibata 1994), etc.), and current sheets that, as we will see, seem to be of fundamental importance to the electrodynamics of the system. The above regions where perfect coupling between particles and the magnetic field breaks down influence the solution of the ideal MHD problem through the boundary conditions. We 
dealt with the presence of particle acceleration gaps in previous papers (Contopoulos 2005; Contopoulos \& Spitkovsky 2006). We show there that particle acceleration along the magnetic field "consumes" a certain amount of electric potential $V_{\text {gap }} \sim 10^{13} \mathrm{~V}$ (e.g. Hibschman \& Arons 2001) from the total "available" electromotive potential

$V=\frac{\Psi_{\text {open }}}{2 \pi r_{\mathrm{lc}}} \sim 10^{13-17}$ Volt

that develops on the surface of the rotating neutron star; therefore, only the remaining amount is available for establishing the global ideal MHD magnetosphere. In general, $V_{\text {gap }} \ll V$, except for pulsars near their death line where $V_{\text {gap }} \sim V$, in which case, the presence of particle acceleration gaps has important implications for the neutron star spindown (see Contopoulos \& Spitkovsky 2006).

In the present work, we deal with the second region where ideal MHD breaks down, namely the global magnetospheric current sheet. In order to understand how reconnection may work in the pulsar magnetosphere, we begin in Sect. 2 with the presentation of a simple magnetostatic analog. In Sect. 3, we argue that reconnection does take place, and in Sect. 4 we show that reconnection extends from the light cylinder to the pulsar wind termination shock, and that it modifies the global structure of the pulsar magnetosphere. In Sect. 5 we discuss the significance of reconnection for the acceleration of the pulsar wind to the low $\sigma_{\text {shock }}$ values inferred by observations. Finally, in Sect. 6 we present a summary of our conclusions.

\section{A simple magnetostatic analog}

The role of current sheets in ideal MHD is to help accomodate the requirements imposed on the system by the specific boundary conditions of the particular problem under investigation. To illustrate our point, let us consider a simple non-relativistic axisymmetric system consisting of a magnetostatic dipole surrounded by an equatorial thin disk of half-thickness

$h \ll r$,

with a hole inside a radius $r=r_{\text {open }}$. The magnetic field structure may be obtained as a solution of the force-free (and current-free outside the disk) problem described by

$\nabla \times \boldsymbol{B}=0$

or, equivalently,

$\frac{\partial^{2} \Psi}{\partial r^{2}}-\frac{1}{r} \frac{\partial \Psi}{\partial r}+\frac{\partial^{2} \Psi}{\partial z^{2}}=0$,

with boundary conditions a) dipolar field at the center and b) horizontal field right above and below the thin disk,

$B_{r}\left(r<r_{\text {open }} ; z=0\right)=0$, and

$B_{z}\left(r \geq r_{\text {open }} ; z=h\right)=B_{z}\left(r \geq r_{\text {open }} ; z=-h\right)=0$.

These particular boundary conditions determine the magnetic field structure shown in Fig. 1, which further implies the presence of a current sheet in the azimuthal direction all along the surface of the infinitely thin disk. Note that, because of the symmetry of the problem, $B_{r}(r ; z)=-B_{r}(r ;-z), B_{\phi}(r ; z)=$ $-B_{\phi}(r ;-z)$, and $B_{z}(r ; z)=B_{z}(r ;-z)$.

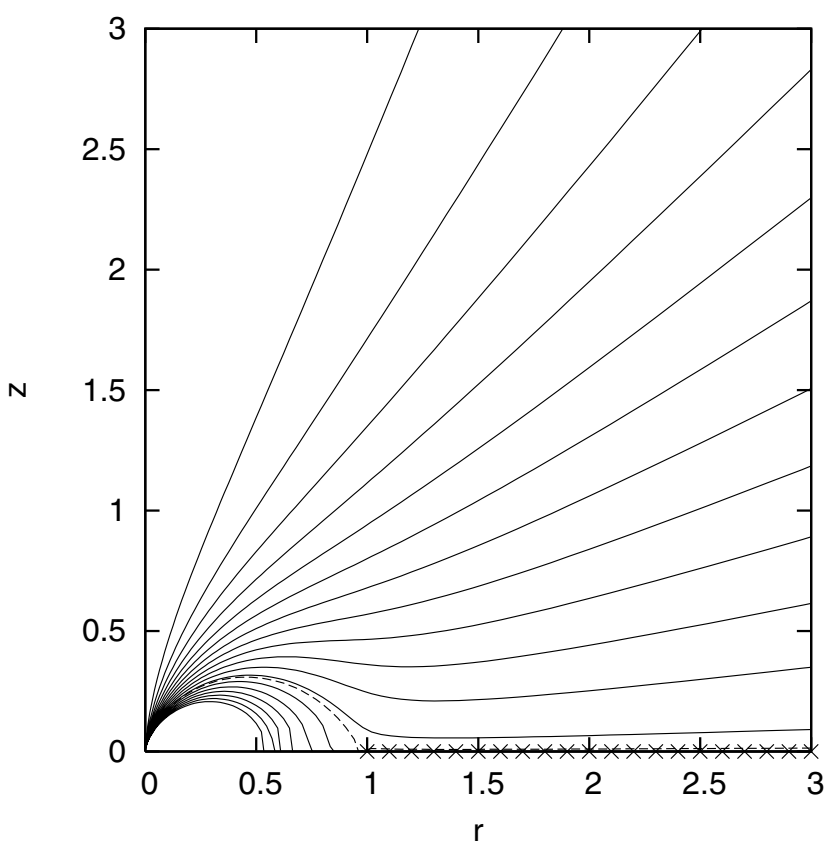

Fig. 1. The poloidal magnetic field structure of a magnetic dipole surrounded by an infinitely conducting thin disk with a hole inside some radius $r_{\text {open }}$. The disk is denoted schematically with a line of x's along the equator. Length units are normalized to $r_{\text {open }}$. Solid lines correspond to magnetic flux intervals of $0.1 \Psi_{\text {dipole }}$, where $\Psi_{\text {dipole }}$ is defined as the amount of magnetic flux of a pure dipole that would cross the equator at distances greater or equal to $r_{\text {open }}$. The dashed line denotes the separatrix $\Psi=\Psi_{\text {open }}=1.23 \Psi_{\text {dipole }}$ between open and closed field lines. There is a close similarity between the present solution and the poloidal structure of the CKF magnetosphere (CKF).

The reader may have already noticed that the field structure obtained in the infinite conductivity case, Fig. 1, is very similar to the poloidal magnetic field structure of the CKF solution ${ }^{1}$. Moreover, one realizes that the assumption of infinite conductivity (i.e. no field reconnection) has been central in obtaining the CKF solution, where we required that field lines open up beyond a certain radius $r_{\text {open }}$, and never again reconnect.

Now, let us assume that, once the solution shown in Fig. 1 is established, we somehow introduce a small amount of resistivity (or equivalently diffusivity) in the disk. Formerly open magnetic field lines will now close across the disk and will slowly begin to diffuse inward. If the diffusion is slow enough, we may approximate the magnetic field evolution as a sequence of force-free equilibria outside the resistive thin disk, coupled to the magnetic field threading the disk. The evolution of the latter is determined by the induction equation inside the disk,

$\dot{\boldsymbol{B}}=-c \nabla \times \boldsymbol{E}=\nabla \times(\boldsymbol{v} \times \boldsymbol{B}-\eta \nabla \times \boldsymbol{B})$.

We have made use here of the generalized Ohm's law

$\boldsymbol{E}+\frac{\boldsymbol{v} \times \boldsymbol{B}}{c}=\frac{\eta}{c} \nabla \times \boldsymbol{B}$,

1 The equatorial boundary conditions, Eqs. (11) and (12), are the same as in the CKF problem (described by Eq. (32) below). Therefore a solution of Eq. (10) "kills" the first part on the 1.h.s. of Eq. (32). Moreover, around and beyond the light cylinder, the r.h.s. balances the second part on the 1.h.s. of Eq. (32). 

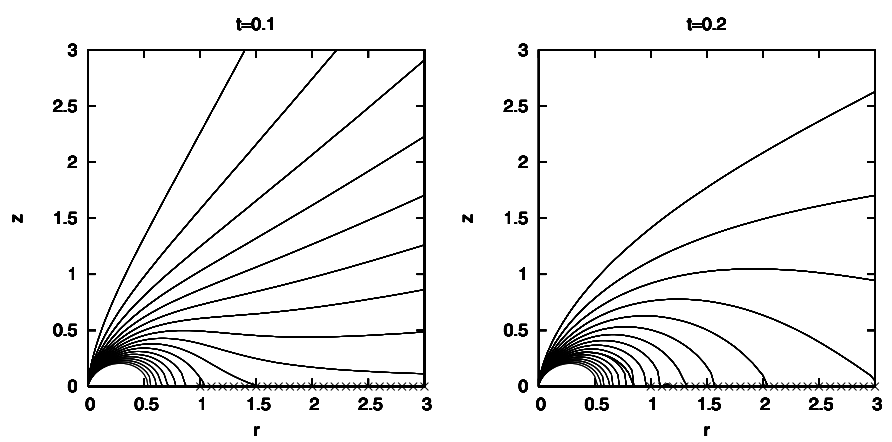

Fig. 2. A typical sequence of poloidal magnetic field configurations when we introduce magnetic diffusivity into the equatorial disk of Fig. 1. Units same as in Fig. 1. Time is normalized to the characteristic timescale $r_{\text {open }} h / \eta$, where $\eta$ and $h \ll r$ are the disk magnetic diffusivity and half thickness, respectively. Note the development of large radial stresses in the interior of the rigid diffusive disk. In the limit $t \rightarrow \infty$, the magnetic field configuration approaches that of a pure magnetostatic dipole, which is a stress-free configuration.

where $v$ and $\eta$ are the matter velocity and magnetic diffusivity in the disk respectively. The z-component of Eq. (13) yields

$\dot{B}_{z}(z=0)=\frac{1}{r} \frac{\partial}{\partial r}\left(-r v_{r} B_{z}-\eta r \frac{\partial B_{r}}{\partial z}\right)$

or, equivalently,

$\dot{\Psi}=-2 \pi r v_{r} B_{z}-2 \pi \eta r \frac{\partial B_{r}}{\partial z} \approx-2 \pi r v_{r} B_{z}-2 \pi \eta r \frac{B_{r}(z=h)}{h}$,

where, we have made use of the fact that the disk is thin, and hence $\partial / \partial z \gg \partial / \partial r$. When a flow field is present in the disk, as is the case for the pulsar magnetosphere, the magnetic flux evolution will be determined by the imbalance between advection and diffusion (first and second terms in the r.h.s. of Eq. (16), respectively). In the present section, we will only consider the simplest case $\boldsymbol{v}=0$. A radial equatorial flow with $\boldsymbol{v} \approx c \hat{\boldsymbol{r}}$ will be introduced in the next section.

One may evolve the above system of equations (Eqs. (10) and (16)), as follows:

1. Start with any initial distribution $B_{z}\left(r \geq r_{\text {open }} ; z=0 ; t=0\right)$ in the disk (in the example discussed here, we take $B_{z}(r \geq$ $\left.\left.r_{\text {open }} ; z=0 ; t=0\right)=0\right)$.

2. Solve Eq. (10) to obtain the field structure above and below the disk. This also yields the distribution $B_{r}\left(r \geq r_{\text {open }} ; z=\right.$ $h ; t=0)$ on the surface of the disk.

3. Obtain the distribution $B_{z}\left(r \geq r_{\text {open }} ; z=0 ; t=t+\mathrm{d} t\right)$ by evolving Eq. (16) for one time step $\mathrm{d} t$.

4. Repeat steps 1 to 3 to obtain the field evolution at all times $t$.

A sequence of field equilibria is shown in Fig. 2, where timescales are normalized to the characteristic time $r_{\text {open }} h / \eta$. One sees that, if we allow for an infinitesimally small amount of magnetic diffusivity in the disk, given enough time, the field structure will approach asymptotically the structure of an isolated magnetostatic dipole (in that case, $B_{r}(z=h) \approx B_{r}(z=0)=$ 0 , and thus, $\dot{\Psi}=0)$. We emphasize that this would not have been possible, had we insisted that the disk were infinitely conductive, as is the case in Fig. 1.

Up to now, we have ignored the large radial magnetic stresses that develop inside the thin disk in the presence of reconnection (see Fig. 2). The infinite conductivity solution shown in Fig. 1 is stress-free and is therefore valid even for a non-rigid disk.
However, in that case the solution would be unstable, and any small perturbation would result in global magnetospheric oscillations. Any small amount of dissipation would damp those oscillations, and the field structure would approach the structure of the isolated magnetostatic dipole asymptotically, which is the structure with the lowest possible magnetic field energy (asymptotically, $B \propto r^{-3}$ for the dipole, and $\propto r^{-2}$ for the solution shown in Fig. 1, respectively). We conclude that the sequence of field equilibria shown in Fig. 2 will take place only if the equatorial disk is "rigid enough" to hold the radial magnetic stress that develops because of the presence of reconnection.

\section{An argument for reconnection}

We now argue that reconnection does indeed take place in the pulsar magnetosphere. We would like to note that, although magnetic reconnection is believed to play a very important role in various systems of physical and astrophysical interest (flares in the solar corona, planetary magnetospheres, laboratory plasmas, etc.), we know very little about the physical processes that control reconnection in other astrophysical systems like pulsar magnetospheres and accretion disks. Although it is customary to assume conditions of infinite conductivity, we will see that this is not justified by observations in the case of the pulsar magnetosphere.

One thing we are certain about is that pulsars spin down. We also believe that the spindown is due to magnetospheric torques. Equating the magnetospheric energy loss (Eq. (5)) to the observed stellar spindown (Eq. (1)), one obtains a relation between $\dot{\Omega}$ and $\Omega$, namely that

$$
\dot{\Omega}=\frac{5}{8} \frac{B_{*}^{2} r_{*}^{4}}{M_{*} c} \frac{\Omega}{r_{\mathrm{open}}^{2}}
$$

Furthermore, in the case of the six pulsars of Table $1, \dot{\Omega}$ is found to be proportional to some power of $\Omega$

$\dot{\Omega} \propto \Omega^{n}$,

where $n$, the braking index, has been observed to have values between 1 and 3 . This implies that

$\frac{r_{\text {open }}}{r_{\mathrm{lc}}}=\left(\frac{\Omega}{\Omega_{0}}\right)^{\frac{3-n}{2}}<1$

(and in fact, in some cases, $r_{\text {open }} / r_{\mathrm{lc}} \ll 1$ ). We have assumed here that $n$ remains unchanged throughout the pulsar's lifetime (a simplification) and that $r_{\mathrm{open}}=r_{\mathrm{lc}}$ at pulsar birth when $\Omega=\Omega_{0}$. If we assume that there is no reconnection, we imply that $\Psi_{\text {open }}$, and consequently $r_{\text {open }}$, remain fixed to the value they attained at pulsar birth. This is equivalent to $\dot{\Omega} \propto \Omega$, and therefore $n=1$, which is not what is observed.

We conclude that in order to account for braking index values $n>1$ in our standard picture of electromagnetic pulsar spindown, reconnection has to take place somewhere in the pulsar magnetosphere outside $r_{\text {open }}$. As we see in the next section, the natural place to look for it is the equatorial current sheet that develops outside $r_{\text {open }}$. We may obtain an estimate of reconnection, by calculating the rate of decrease in the open magnetic flux as the pulsar spins down,

$\dot{\Psi}_{\text {open }}=\frac{(n-1)}{2} \Psi_{\text {open }} \frac{\dot{\Omega}}{\Omega} \sim-10^{-3} \frac{\Psi_{\text {open }}}{\text { year }}$ 
Table 1. Characteristic parameters for the 6 pulsars with measured values of the braking index $n$.

\begin{tabular}{lccccccc}
\hline \hline & $n$ & $\frac{\eta}{h c}^{a}$ & $\begin{array}{c}r_{\text {open }}{ }^{b} \\
\left(10^{8} \mathrm{~cm}\right)\end{array}$ & $\begin{array}{c}r_{\mathrm{lc}} \\
\left(10^{8} \mathrm{~cm}\right)\end{array}$ & $\begin{array}{c}\frac{\Psi_{\text {open }}}{\mid \dot{\Psi}_{\text {open }}} \\
\left(10^{3} \text { years }\right)\end{array}$ & $\sigma_{\text {shock }}{ }^{c}$ & $\frac{L_{\text {field }}}{L_{\text {particles }}}$ \\
\hline Crab & 2.515 & 0.08 & 1.2 & 1.6 & 3 & $10^{-2}-10^{-3}$ & $8 \times 10^{-3}$ \\
Vela & 1.4 & 0.02 & 0.7 & 4.3 & 113 & $1-10^{-1}$ & $7 \times 10^{-1}$ \\
PSR 1509-58 & 2.84 & 0.09 & 5.8 & 7.2 & 3 & & $4 \times 10^{-3}$ \\
PSR B0540-69 & 2.140 & 0.06 & 1.2 & 2.4 & 6 & & $4 \times 10^{-2}$ \\
PSR J1846-0258 & 2.65 & 0.08 & 8.4 & 15.5 & 2 & & $8 \times 10^{-3}$ \\
PSR J1119-6127 & 2.91 & 0.10 & 16.5 & 19.5 & 3 & & $4 \times 10^{-3}$ \\
\hline
\end{tabular}

${ }^{a} \eta / h c=0.05(n-1) ;{ }^{b}$ we assumed an initial period of $1 \mathrm{~ms}$ at pulsar birth; ${ }^{c} r_{\text {shock }} \sim 0.1 \mathrm{pc}$.

(see Table 1). We would like to emphasize here that $\dot{\Psi}_{\text {open }} \neq 0$ has nothing to do with the pulsar spinning down. Even in the theoretical case where some artificial source of energy kept $\Omega$ unchanged, as long as $r_{\mathrm{open}}<r_{\mathrm{lc}}$ the closed line region would grow against the open line region at a rate determined by Eq. (16). This realization allows us to relate the magnetic diffusivity in the equatorial current sheet immediately outside $r_{\text {open }}$ to the observable pulsar parameters, namely that

$\left.\frac{\eta}{h c} \sim \frac{|\dot{\Psi}|}{2 \pi r r_{\mathrm{lc}} \Omega B_{r}(r ; z=h)}\right|_{r=r_{\mathrm{open}}^{+}}+\left.\frac{\left|B_{z}\right|}{B_{r}(r ; z=h)}\right|_{r=r_{\mathrm{open}}^{+}}$.

We have introduced here a radial equatorial flow with $v_{r} \approx c$ beyond $r=r_{\text {open }}$. This accounts for the high Lorentz factor electron-positron outflow that is expected to flow along the open magnetic field lines. Inside the corrotating closed line region $B_{r}(z=0)=0$ (see CKF), and therefore just outside $r_{\text {open }}$, $B_{r}(z=h)$ is also very small (Fig. 11, in Timokhin 2006). Moreover, in the ideal MHD case $(\mathrm{CKF}), B_{z}(z=0)$ is taken to be equal to zero beyond $r_{\text {open }}$. In the presence of reconnection, we expect that $\left|B_{z}\right| \ll\left|B_{r}\right|$ in the equatorial region beyond $r_{\text {open }}$ (see solution in the next section), therefore, we may ignore the second term in the r.h.s. of Eq. (21). We thus propose a simple model where $\eta / h c$ only depends on the braking index $n$ through the following approximate relation,

$\frac{\eta}{h c}=\kappa(n-1)$

where the parameter $\kappa$ will be determined in Sect. 5 . This model is consistent with our understanding that, the further $n$ differs from unity, the greater the role of reconnection in the magnetosphere.

We would like to remind the reader that "a braking index that reflects the physics of the pulsar braking mechanism can only be measured for the very youngest pulsars which show either little glitch activity or have been observed for many years, in which case the statistical effect of glitches can be determined" (Hobbs et al. 2004). This is why we have restricted our discussion to the case of the six pulsars of Table 1 . However, in view of the recent measurements of $\ddot{\Omega}$ for many more pulsars (e.g. Johnston \& Galloway 1999; Hobbs et al. 2004), one might be tempted to also consider values of $n$ very different from the ones in Table 1. In the context of our present model, values of $n \gg 1$ may be interpreted as a closed line region growing through very efficient (anomalous?) reconnection $\sim n$ times faster than the dipole canonical value ${ }^{2}$. On the other hand, values of $n \ll 1$ may be obtained when a strong stellar wind acts to open up formerly closed

\footnotetext{
${ }^{2}$ Other physical mechanisms such as magnetic field decay (e.g. Urpin \& van Riper 1993) may also be responsible for higher than normal values of $n$.
}

magnetic field lines (e.g. Harding et al. 1999). In this picture, reconnection is irrelevant. One may even speculate that the wide range of $n$ values may reflect a long period (100-1000 years) cyclic evolution of the system between the above two extremes (see Beskin et al. 2006).

\section{Continuous equatorial reconnection}

In the previous section, we only considered reconnection in the poloidal magnetic field component, which is generated by a corresponding toroidal electric current. The MHD rotator, however, also develops a toroidal magnetic field component $B_{\phi}$ with a corresponding poloidal electric current (Eq. (4)) such that

$B_{\phi}=\frac{2 I(\Psi)}{c r}$

The most natural place one would expect reconnection to take place is the global magnetospheric current sheet where the electric current density is the greatest. We remind the reader that an important element of the force-free ideal MHD solution is the presence of an equatorial current sheet (poloidal + toroidal) that extends from the surface of the star, along the separatrix between open and closed field lines and along the equator. The poloidal component of this current sheet constitutes the return current for the globally distributed (i.e. non-singular) electric current that flows along the open field lines from the stellar surface to infinity. This particular electric current distribution, and therefore the corresponding return current sheet, was obtained as an eigenvalue of the problem by requiring that there is no magnetic field discontinuity at the mathematical singularity of the light cylinder. We would like to emphasize this point again: given the particular equatorial boundary condition that we have chosen in CKF, namely that $B_{z}\left(r>r_{\text {open }} ; z=0\right)=0$, the existence of the above global current sheet is inevitable.

As we now see, the latter choice of equatorial boundary condition is not $100 \%$ valid when we introduce nonzero magnetic diffusivity $\eta$ and half thickness $h$ in the equatorial poloidal current sheet. Because of the symmetry of the problem,

$\boldsymbol{B}(r ; z=0)=B_{z} \hat{\boldsymbol{z}}$,

and therefore, when $B_{z}(z=0) \neq 0$ a radial component of the magnetospheric poloidal electric field $\boldsymbol{E} \equiv-r \Omega \hat{\phi} \times \boldsymbol{B}_{\mathrm{p}} / c$ develops along the surface of the equatorial current sheet, namely

$E_{r}(r ; z=h)=-\frac{\Omega r}{c} B_{z}(r ; z=h) \approx-\frac{\Omega r}{c} B_{z}(r ; z=0)$.

For a slowly evolving magnetosphere, the $\phi$-component of the induction equation for the equatorial current sheet (Eq. (13)) implies that

$\left.\frac{\partial E_{r}}{\partial z}\right|_{z \sim 0} \approx 0$, 
which further implies that $E_{r}(z=h) \approx E_{r}(z=0)$ or, equivalently

$\Omega r B_{z} \approx \eta \frac{\partial B_{\phi}}{\partial z} \approx \frac{\eta B_{\phi}(z=h)}{h}=\frac{\eta}{h c} \frac{2 I(\Psi)}{r}$.

This may be rewritten as

$\frac{\partial \Psi}{\partial r} \approx \frac{\eta}{h c} \frac{4 \pi I(\Psi)}{\Omega r}$

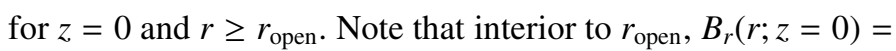
0 , and therefore the field structure is close to dipolar with

$\frac{\partial \Psi}{\partial r} \approx-\frac{\Psi}{r}$.

Here, $I(\Psi)$ is determined similarly to CKF as an eigenvalue of the solution of the force-free magnetosphere outside the current sheet (see below).

The physical meaning of Eq. (27) is that the vertical ( $z$ ) component of the electromagnetic (Poynting) energy flux is nonzero at the surface of the equatorial current sheet,

$\frac{c}{4 \pi} \boldsymbol{E} \times\left.\boldsymbol{B}\right|_{z}=\frac{c}{4 \pi} E_{r} B_{\phi}(z=h)$

hence electromagnetic energy is continuously channeled to the equatorial current sheet where it is transformed into some different (non-electromagnetic) form. As we see in the next section, it is transformed into particle kinetic energy. Another way to look at Eq. (27) is that field lines "corotate" with the neutron star at angular velocity $\Omega$, so that particles "trapped" along field lines would corotate at speeds $r \Omega$ (and at distances $r>r_{\mathrm{lc}}$ these would exceed the speed of light) unless the equatorial region is diffusive enough for the field line to "slip through" the particles at a rate given by the r.h.s. of Eq. (27). This is a generalization of the argument that led us in CKF to propose the boundary condition that $B_{z}\left(r>r_{\mathrm{lc}} ; z=0\right)=0$.

If one knows the distribution of $\eta / h$ with distance along the equatorial current sheet, Eq. (28), together with the special relativistic generalization of Eq. (9),

$(\nabla \times \boldsymbol{B}) \times \boldsymbol{B}+4 \pi \rho_{\mathrm{e}} \boldsymbol{E}=0$,

or equivalently

$\left(1-\frac{r^{2}}{r_{\mathrm{lc}}^{2}}\right)\left(\frac{\partial^{2} \Psi}{\partial r^{2}}-\frac{1}{r} \frac{\partial \Psi}{\partial r}+\frac{\partial^{2} \Psi}{\partial z^{2}}\right)+\frac{2}{r} \frac{\partial \Psi}{\partial r}=-\frac{4 I(\Psi)}{c^{2}} \frac{\mathrm{d} I(\Psi)}{\mathrm{d} \Psi}$,

yield the structure of the pulsar magnetosphere all the way to the wind termination shock distance $r_{\text {shock }} \sim 10^{9} r_{\text {open }}$. We may expect that the presence of equatorial reconnection modifies the CKF solution only slightly, with magnetic flux gradually reconnecting along the equator.

In order to obtain a solution for the structure of the global pulsar magnetosphere, we make the simplification that $\eta / h$ remains constant at all distances, equal to the value given by Eq. (22). We argued that, for low enough values of $\eta / h$, the structure of the magnetosphere will not differ much from that of the CKF solution, and in particular, the poloidal electric current distribution will not differ much from Eq. (4). In that case, Eq. (28) yields

$\Psi=\Psi_{\text {open }} \frac{2}{1+\left(\frac{r}{r_{\text {open }}}\right)^{2 \kappa(n-1)}}$

for $z=0$ and $r \geq r_{\text {open. }}$. Given the equatorial distribution of $\Psi$, we obtain the global magnetospheric structure shown in

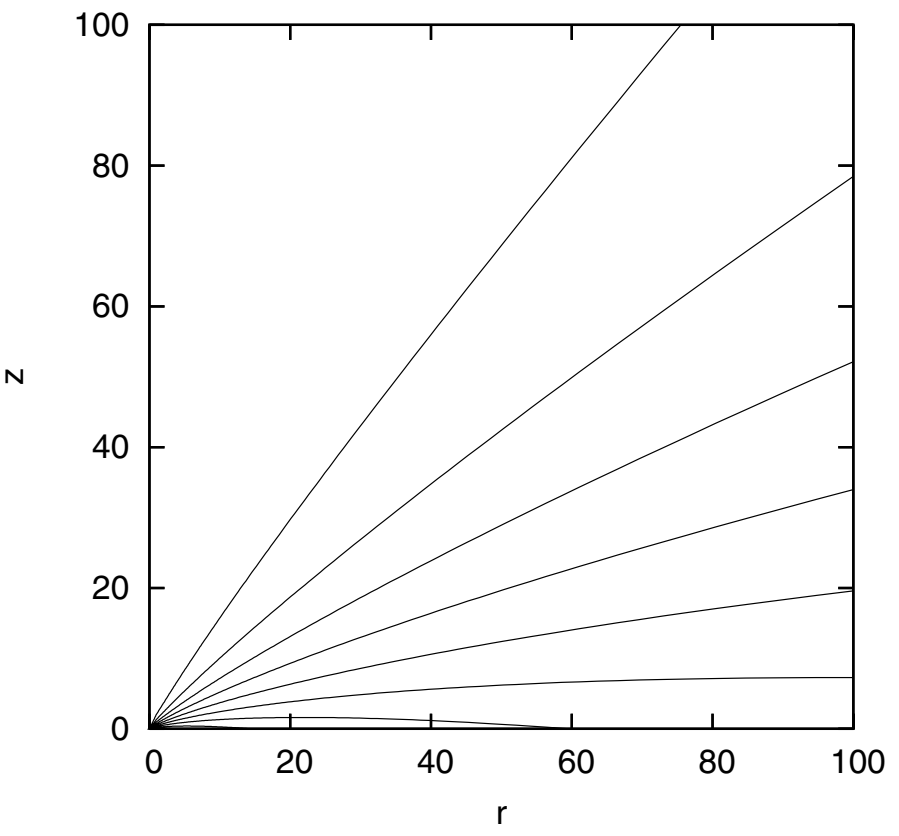

Fig. 3. The modified structure of the large-scale axisymmetric pulsar magnetosphere when we account for magnetic diffusivity in the equatorial current sheet. Units same as in Fig. 1 . We set $\eta / h=0.08 c$, a value that we believe is relevant for the Crab pulsar magnetosphere (see text). One sees the closing of the magnetosphere across the equatorial current sheet. Note that an amount of poloidal magnetic flux equal to about $0.3 \Psi_{\text {open }}$ reconnects across the equatorial current sheet inside about $100 r_{\mathrm{lc}}$.

Figs. 3 and 4 for 2 different values of $n$. Note that we have assumed here that, since reconnection is proportional to the electric current density, the perfect particle-field coupling required in deriving Eq. (32) remains valid everywhere throughout the magnetosphere, except in regions with very large electric current density, as is the case for the equatorial current sheet.

We are thus in a position to estimate the amount of magnetic flux that will close inside the pulsar wind termination shock as a function of the diffusivity parameter $\eta / h c$ and, more specifically, as a function of the difference $(n-1)$. As we see in the next section, this result may be related to the acceleration mechanism in the pulsar wind.

\section{Equatorial reconnection and wind acceleration}

We have shown that the small amount of reconnection implied by the observations of pulsar spindown requires the presence of a radial electric field component along the equatorial return current sheet in the pulsar magnetosphere (Eq. (25)). In other words, a potential drop

$V_{\mathrm{rec}}=\left(1-\frac{\Psi\left(r_{\text {shock }}\right)}{\Psi_{\text {open }}}\right) V$

develops along the equatorial current sheet, from about the light cylinder to the distance of the pulsar wind termination shock. Here, $\Psi\left(r_{\text {shock }}\right)$ is the amount of magnetic flux that does not close across the equatorial current sheet by the time the pulsar wind reaches the termination shock.

According to reconnection theory (e.g. Priest \& Forbes 2000), when the value of the reconnection electric field (in our 


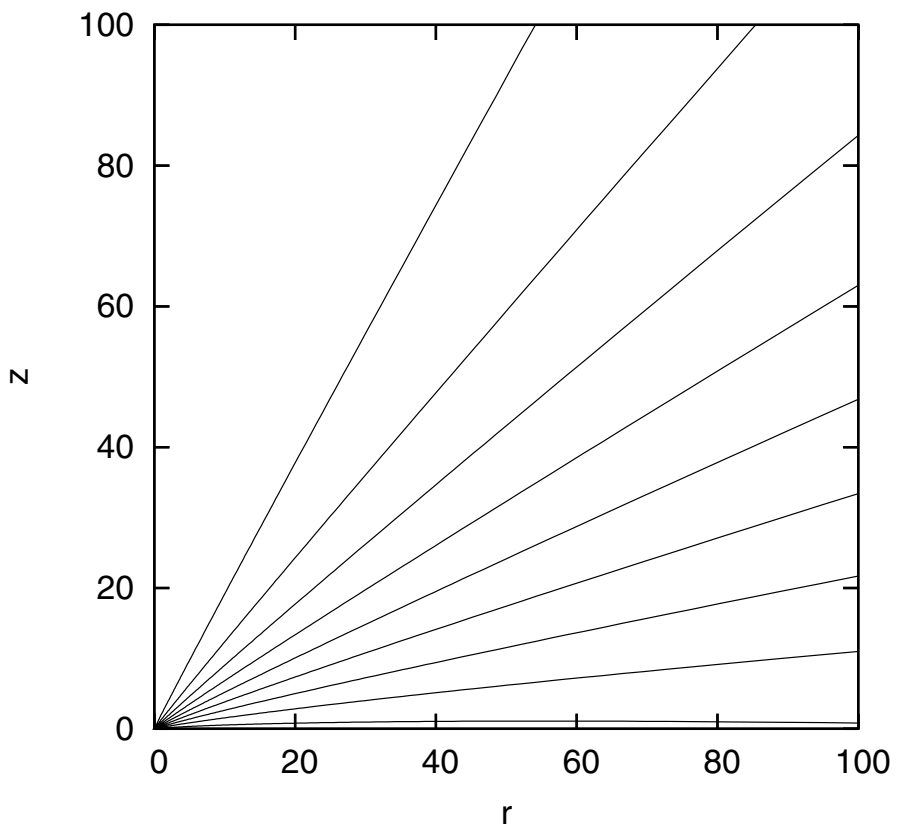

Fig. 4. Same as Fig. 3 with $\eta / h=0.02 c$, a value that we believe is relevant for the Vela pulsar magnetosphere (see text). The closing of the magnetosphere across the equatorial current sheet is slower when the current sheet magnetic diffusivity is smaller. Note that the amount of poloidal magnetic flux that reconnects inside about $100 r_{\mathrm{lc}}$ is equal to about $0.1 \Psi_{\text {open }}$, i.e. smaller than in Fig. 3 .

case $E_{r}$ as given in Eq. (25)) is greater than a characteristic value, the Dreicer field defined as

$E_{D}=\frac{2 \pi e^{3} n_{\mathrm{e}} \ln \Lambda}{k T_{\mathrm{e}}}$

particles that enter the current sheet get accelerated by the electric field and do not interact during their acceleration process. Here, $n_{\mathrm{e}}$ and $T_{\mathrm{e}}$ are the particle number density and temperature in the current sheet, respectively, and $\ln \Lambda$ is the Coulomb logarithm. For number densities up to a few orders of magnitude greater than the local Goldreich-Julian number density (Goldreich \& Julian 1969), the radial electric field inside the pulsar magnetosphere equatorial current sheet is found to be orders of magnitude larger than the Dreicer field, as is the case in the geomagnetic tail (see Lyons \& Williams 1984, for a review). In this situation, the orbits of individual particles must be calculated by explicit integration of their equations of motion, and the acceleration mechanism is essentially a finite gyroradius effect (i.e. non-MHD). According to that scenario, energy in the magnetic field lines that reconnect across the current sheet may be transformed into the kinetic energy of the accelerated particles without dissipation into other forms of energy, (Speiser (1987) refers to this process as "inertial dissipation"). This result is quite encouraging. The equatorial potential drop (Eq. (34)) is available to accelerate some particles (electrons/positrons) up to Lorentz factors

$\Gamma_{\text {shock }}=\frac{e V}{m_{\mathrm{e}} c^{2}}\left(1-\frac{\Psi\left(r_{\text {shock }}\right)}{\Psi_{\text {open }}}\right) \sim\left(1-\frac{\Psi\left(r_{\text {shock }}\right)}{\Psi_{\text {open }}}\right) 10^{11}$.

We have obtained here a perfect particle accelerator, all the way from the light cylinder to the termination shock. It thus seems natural to associate the particle-dominated wind that is observationally inferred in the case of the Crab pulsar (Rees \& Gunn 1974; Kennel \& Coroniti 1984), with the particles that belong to the equatorial current sheet (mainly positrons/electrons for an aligned/counter-aligned rotator, respectively). In a realistic pulsar, the finite angle between the magnetic and rotation axes, and the finite scale height $h$ of the equatorial current sheet will lead to a finite opening of the equatorial particle dominated pulsar wind. Note that our picture of gradual poloidal + toroidal magnetic field reconnection is different from the reconnection of the toroidal magnetic field that has been proposed as a source of the pulsar wind acceleration in a "stripped wind" (Coroniti 1990; Michel 1994; Lyubarsky \& Kirk 2001).

We have started to investigate the relativistic dissipationless acceleration of particles in the equatorial current sheet (Contopoulos, in preparation). This by itself is a very promising new area of research. The dynamics of the equatorial current sheet will also be studied. In particular, in analogy to the issue of the rigidity of the conducting disk in our simple magnetostatic analog of Sect. 2, we need to address the issue of the stability of the current sheet in the presence of the radial magnetic stresses that develop across it due to reconnection. It is interesting that the ideal MHD CKF solution implies that the current sheet is electrically charged (the vertical component of the electric field on the surface of the current sheet, $E_{z}=\Omega r B_{r} / c$, changes sign across it), and therefore, we expect that the radial electric field $E_{r}$ that develops along it contributes to counteract the radial magnetic stress. Note that dissipationless particle acceleration due to reconnection in the equatorial current sheet was first discussed in Romanova et al. (2005). Our presentation, however, differs from theirs in that we tried to relate the toroidal magnetic field reconnection to the global redistribution of the poloidal magnetic field in the pulsar magnetosphere. This may also be seen from the fact that our equatorial radial electric field scales as $\left(r / r_{\mathrm{lc}}\right)^{-1-\epsilon}$ with $\epsilon \ll 1$, whereas in their work it drops much faster with distance as $\left(r / r_{\mathrm{lc}}\right)^{-2}$.

We conclude here with a discussion of the overall energy balance in the pulsar magnetosphere. In our simple picture of constant $\eta / h$, we may estimate the fraction of the total spindown energy flux that remains in the from of electromagnetic field energy by the time we reach the pulsar wind termination shock,

$$
\begin{aligned}
\frac{L\left(\Psi\left(r_{\text {shock }}\right)\right)}{L\left(\Psi_{\text {open }}\right)} & \approx \frac{\int_{\Psi=0}^{\Psi\left(r_{\text {shock }}\right)} I(\Psi) \mathrm{d} \Psi}{\int_{\Psi=0}^{\Psi_{\text {open }}} I(\Psi) \mathrm{d} \Psi} \\
& =\frac{3}{2}\left(\frac{\Psi\left(r_{\text {shock }}\right)}{\Psi_{\text {open }}}\right)^{2}\left[1-\frac{1}{3} \frac{\Psi\left(r_{\text {shock }}\right)}{\Psi_{\text {open }}}\right] \\
& \sim 2\left[1+3\left(\frac{r_{\text {shock }}}{r_{\text {open }}}\right)^{2 \kappa(n-1)}\right] \cdot\left[1+\left(\frac{r_{\text {shock }}}{r_{\text {open }}}\right)^{2 \kappa(n-1)}\right]^{-3}
\end{aligned}
$$

(e.g. Gruzinov 2005). The rest of the spindown energy flux is gradually channeled to the particles along the equatorial sheet. It is very tempting to associate the inferred values of $\sigma_{\text {shock }}$ (defined as the ratio of energy flux in the electromagnetic field to the energy flux in the particles at the pulsar wind termination shock), with the energy flux estimated above remaining in the form of electromagnetic field energy to the energy flux transferred to the particles in the equatorial current sheet. It is interesting that by fixing our only free parameter to the value

$$
\kappa=0.05
$$

we are able to produce estimates for both the Crab and Vela observationally inferred values of $\sigma_{\text {shock }}$ (see Table 1 ). 


\section{Summary and conclusions}

We have presented here a new picture for the global structure of the pulsar magnetosphere, where field lines remain closed even beyond the light cylinder (Figs. 3 and 4). This is only possible if we take into account the effect of magnetic field reconnection across the equatorial magnetospheric current sheet. We argue that observations of braking index values $n>1$ allow us to infer that reconnection is indeed at work in the pulsar magnetosphere, so we produce an estimate of the reconnection time scale (Eq. (20); Table 1). In fact, we propose to quantify the amount of effective magnetic diffusivity as being proportional to the difference $(n-1)$ (Eqs. (21) and (22)). In our model, the neutron star spindown energy carried in the form of electromagnetic Poynting flux is gradually directed towards the equator where it is channeled to the particles along the equatorial sheet with no radiation losses (Eq. (37)). Without considering the details of dissipationless particle acceleration, we argued that individual particles may reach very high Lorentz factors (Eq. (36)). Finally, we discussed the issue of the very low $\sigma_{\text {shock }}$ values obtained at the pulsar wind termination shock. In our picture the regions of field and particle-dominated flow are physically separated, and we can estimate the ratio of the respective energy fluxes. If then we assume that these regions somehow mix at the termination shock, we are able to estimate the values of $\sigma_{\text {shock }}$. When we calibrate our diffusivity parameter (Eq. (22)) in order to obtain a $\sigma_{\text {shock }}$ value relevant to the Crab pulsar wind termination shock, all $\sigma_{\text {shock }}$ values lie in the range $10^{-2}-10^{-3} e x$ cept for the Vela pulsar where $\sigma_{\text {shock }}$ is believed to have a value close to unity. Thus, the Vela pulsar is singular, because it has a much higher value of $\sigma_{\text {shock }}$, together with a much lower value of the braking index $n$. Our present work offers a physical picture where these two observed parameters are related. It is interesting that Vela is also singular in that it shows a very strong glitch activity. This may contribute to a more effective loading of the magnetosphere with charges, and thus explain the reduced effective magnetic diffusivity in the equatorial current sheet.

We conclude that collisionless relativistic reconnection along the equatorial current sheet may play a fundamental role in determining both the near and large-scale structure of the pulsar magnetosphere, and in accounting for the acceleration of the pulsar wind; it therefore certainly deserves further investigation.

Acknowledgements. We would like to thank Roger Romani and Anatoly Spitkovsky for their strong criticism that led us to reconsider certain parts of the present work. We would also like to thank Christos Efthymiopoulos and Constantinos Gontikakis for introducing us to the field of collisionless reconnection.

\section{References}

Beskin, G., Biryukov, A., \& Karpov, S. 2006 [arXiv: astro-ph/0603375] Bogovalov, S. 1991, A\&A, 349, 1017

Bogovalov, S., \& Tsinganos, K. 1999, MNRAS, 305, 211

Contopoulos, I. 2005, A\&A, 442, 579

Contopoulos, I. 2006, in 363rd Heraeus seminar on Neutron Stars and Pulsars, MPE-Report, No. 291

Contopoulos, I., \& Spitkovsky, A. 2006, ApJ, 643, 1139

Contopoulos, I., Kazanas, D., \& Fendt, C. 1999, ApJ, 511, 351

Coroniti, F. V. 1990, ApJ, 349, 538

Daugherty, J. K., \& Harding, A. K. 1982, ApJ, 252, 337

Goldreich, P., \& Julian, W. H. 1969, ApJ, 157, 869

Gruzinov A. 2005, Phys. Rev. Lett., 94

Harding, A. K., \& Muslimov, A. 2005, Ap\&SS, 297, 63

Harding, A. K., Contopoulos, I., \& Kazanas, D. 1999, ApJ, 525, 125

Hibschman, J. A., \& Arons, J. 2001, ApJ, 554, 624

Hobbs, G., Lyne, A. G., Kramer, M., Martin, C. E., \& Jordan, C. 2004, MNRAS, 353,1311

Johnston, S., \& Galloway, D. 1999, MNRAS, 306, L50

Kennel, C. F., \& Coroniti, F. V. 1984, ApJ, 283, 6994

Lyons, L. R., \& Williams, D. J. 1984, Quantitative Aspects of Magnetospheric Physics (Dordrecht: Reidel)

Lyubarsky, Y., \& Kirk, J. G. 2001, ApJ, 547, 437

Mestel, L. 1999, Stellar Magnetism (Oxford: Clarendon)

Mestel, L., \& Shibata, S. 1994, MNRAS, 271, 621

Michel, F. C. 1991, Theory of Neutron Star Magnetospheres (Chicago: Univ. Chicago Press)

Michel, F. C. 1994, ApJ, 431, 397

Priest, E., \& Forbes, T. 2000, Magnetic Reconnection (Cambridge: UK)

Rees, M. J., \& Gunn, J. F. 1974, MNRAS, 167, 1

Romani, R. W., \& Yadigaroglu, I.-A. 1995, ApJ, 438, 314

Romanova, M. M., Chulsky, G. A., \& Lovelace, R. V. E. 2005, ApJ, 630, 1020

Speiser, T. W. 1987, Phys. Scri. T, 18, 119

Spitkovsky, A. 2006, ApJ, 648, L51

Timokhin, A. N. 2006, MNRAS, 368, 1055

Urpin, V. A., \& van Riper, K. A. 1993, ApJ, 411, L87 